PROCEEDINGS OF THE

AMERICAN MATHEMATICAL SOCIETY

Volume 126, Number 8, August 1998, Pages 2433-2441

S 0002-9939(98)04454-2

\title{
INTEGRATION ON A CONVEX POLYTOPE
}

\author{
JEAN B. LASSERRE
}

(Communicated by David Sharp)

\begin{abstract}
We present an exact formula for integrating a (positively) homogeneous function $f$ on a convex polytope $\Omega \subset R^{n}$. We show that it suffices to integrate the function on the $(n-1)$-dimensional faces of $\Omega$, thus reducing the computational burden. Further properties are derived when $f$ has continuous higher order derivatives. This result can be used to integrate a continuous function after approximation via a polynomial.
\end{abstract}

\section{INTRODUCTION}

We consider the integration of a continuous (positively) homogeneous function $f: R^{n} \rightarrow R$ on a convex polytope $\Omega \subset R^{n}$. We prove that if $f$ is continuously differentiable, it suffices to integrate the function on the $(n-1)$-dimensional faces of $\Omega$. As a continuous function on a compact set in $R^{n}$ can be uniformly approximated by a polynomial (a sum of homogeneous functions), this result provides an alternative method for integrating continuous functions on a convex polytope.

A similar result also holds for an exponential $e^{\langle c, x\rangle}$. In fact, it has even been shown in [1], [2] that it suffices to evaluate that function at the vertices of $\Omega$. This result was then used for computing the volume and counting integral points in $\Omega$.

When $f$ is twice continuously differentiable, one may proceed further, and we show that it suffices to integrate $f$ on the $(n-2)$-dimensional faces and its derivatives on the $(n-1)$-dimensional faces. One may iterate the process when $f$ has higher order continuous derivatives, etc.

\section{Integration OF A HOMOGENEOUS FUNCTION}

Let $A$ be an $(m, n)$-real matrix, $f: R^{n} \rightarrow R$ a real continuous (positively) homogeneous function of degree $q$, i.e. $f(\lambda x)=\lambda^{q} f(x)$ for all $\lambda>0, x \in R^{n}$. For a (positively) homogeneous function of degree $q$ that is continuously differentiable, Euler's formula holds (cf. [5]), i.e.:

$$
q f(x)=\langle\nabla f(x), x\rangle \text { for all } x .
$$

Let

$$
h(b):=\int_{\Omega} f(x) d x \text { with } \Omega:=\left\{x \in R^{n} \mid A x \leq b\right\} .
$$

Received by the editors August 5, 1996 and, in revised form, January 6, 1997.

1991 Mathematics Subject Classification. Primary 65D30.

Key words and phrases. Numerical integration in $R^{n}$, homogeneous functions, convex polytopes. 
We assume that $\Omega$ is a convex polytope. The following fact is straightforward:

Proposition 2.1. If $f$ is (positively) homogeneous of degree $q$, then $h$ is (positively) homogeneous of degree $n+q$.

Proof. We have

$$
h(\lambda b):=\int_{A x \leq \lambda b} f(x) d x=\int_{A(x / \lambda) \leq b} \lambda^{q} f(x / \lambda) \lambda^{n} d(x / \lambda)=\lambda^{n+q} \int_{\Omega} f(x) d x,
$$

which yields the desired result.

Let $\Omega_{i}:=\left\{x \in R^{n} \mid A x \leq b, A_{i}^{T} x=b_{i}\right\}$, i.e. $\Omega_{i}$ is the $(n-1)$-dimensional face of $\Omega$ determined by the hyperplane $A_{i}^{T} x=b_{i}$, where $A_{i}^{T}$ is the $i t h$ row of the matrix $A$. Let $\mathcal{H}_{i}$ denote the $(n-1)$-dimensional affine variety that contains $\Omega_{i}$. The algebraic distance from the point $x_{0}$ to $\mathcal{H}_{i}$ is denoted $d\left(x_{0}, \mathcal{H}_{i}\right)$, and $d\left(x_{0}, \mathcal{H}_{i}\right)=\left(b_{i}-A_{i}^{T} x_{0}\right) /\left\|A_{i}\right\|$ (with $\|$.$\| the usual Euclidean norm). Let \mu$ be the Lebesgue measure on $\mathcal{H}_{i}$. The $n$-dimensional (resp. $(n-1)$-dimensional) volume of $\Omega\left(\right.$ resp. $\left.\Omega_{i}\right)$ is denoted by $\mathcal{V}_{n}(\Omega)$ (resp. $\mathcal{V}_{n-1}\left(\Omega_{i}\right)$ ).

Lemma 2.2. Assume that $f$ is continuously differentiable, $\mathcal{V}_{n}(\Omega) \neq 0$, and $\mathcal{V}_{n-1}\left(\Omega_{i}\right) \neq 0$. Then, $h$ is continuously differentiable at $b$ and

$$
\frac{\partial h}{\partial b_{i}}=\frac{1}{\left\|A_{i}\right\|} \int_{\Omega_{i}} f d \mu
$$

where $\mu$ is the Lebesgue measure on $\mathcal{H}_{i}$, the $(n-1)$-dimensional affine variety that contains $\Omega_{i}$.

Proof. The proof is similar to the proof in [4] for the volume of $\Omega$, i.e. when $f(x) \equiv 1$. For $\delta b_{i}>0$, let $\Delta\left(\delta b_{i}\right)$ be the set

$$
\Delta\left(\delta b_{i}\right):=\left\{x \in R^{n} \mid b_{i} \leq A_{i}^{T} x \leq b_{i}+\delta b_{i}, A_{j}^{T} x \leq b_{j}, j \neq i\right\} .
$$

Since $\mathcal{V}_{n-1}\left(\Omega_{i}\right) \neq 0, \Delta\left(\delta b_{i}\right) \neq \emptyset$ for $\delta b_{i}$ sufficiently small. Consider the change of variables $x=x_{0}+z A_{i} /\left\|A_{i}\right\|+\sum_{j=1}^{n-1} y_{j} v_{j}$, where $A_{i}^{T} x_{0}=b_{i}$ and the $v_{j}$ form an orthonormal basis of the $(n-1)$-dimensional subspace $A_{i}^{T} x=0$. Equivalently, $\Delta\left(\delta b_{i}\right)$ can be written

$$
\begin{aligned}
0 \leq z\left\|A_{i}\right\| & \leq \delta b_{i}, \\
\sum_{k=1}^{n-1} y_{k} A_{j}^{T} v_{k} & \leq b_{j}-A_{j}^{T} x_{0}-z A_{j}^{T} A_{i} /\left\|A_{i}\right\|, j \neq i .
\end{aligned}
$$

Let

$$
s_{j}:=\max \left[0, \frac{\delta b_{i}}{\left\|A_{i}\right\|^{2}} A_{j}^{T} A_{i}\right], s_{j}^{\prime}:=\max \left[0, \frac{-\delta b_{i}}{\left\|A_{i}\right\|^{2}} A_{j}^{T} A_{i}\right], j \neq i,
$$

and let $\Delta^{1}\left(\delta b_{i}\right)$ and $\Delta^{2}\left(\delta b_{i}\right)$ be the domains in $R^{n}$, defined respectively by

$$
0 \leq z \leq \frac{\delta b_{i}}{\left\|A_{i}\right\|}, \sum_{k=1}^{n-1} y_{k} A_{j}^{T} v_{k} \leq b_{j}-A_{j}^{T} x_{0}+s_{j}^{\prime}, j \neq i,
$$

and

$$
0 \leq z \leq \frac{\delta b_{i}}{\left\|A_{i}\right\|}, \sum_{k=1}^{n-1} y_{k} A_{j}^{T} v_{k} \leq b_{j}-A_{j}^{T} x_{0}-s_{j}, j \neq i .
$$


Obviously, $\Delta^{2}\left(\delta b_{i}\right) \subseteq \Delta\left(\delta b_{i}\right) \subseteq \Delta^{1}\left(\delta b_{i}\right)$. Define also

$$
\Delta^{\prime 1}\left(\delta b_{i}\right):=\left\{y \in R^{n-1} \mid \sum_{k=1}^{n-1} y_{k} A_{j}^{T} v_{k} \leq b_{j}-A_{j}^{T} x_{0}+s_{j}^{\prime}, j \neq i\right\},
$$

and

$$
\Delta^{\prime 2}\left(\delta b_{i}\right):=\left\{y \in R^{n-1} \mid \sum_{k=1}^{n-1} y_{k} A_{j}^{T} v_{k} \leq b_{j}-A_{j}^{T} x_{0}-s_{j}, j \neq i\right\} .
$$

Note that $\Delta^{\prime 1}(0)=\Delta^{\prime 2}(0)=\Omega_{i}$.

Assume first that $f$ is nonnegative. From $h\left(b+\delta b_{i} e_{i}\right)-h(b)=\int_{\Delta\left(\delta b_{i}\right)} f d x$, we have

$$
\begin{aligned}
& \int_{0}^{\delta b_{i} /\left\|A_{i}\right\|} \int_{\Delta^{\prime 2}\left(\delta b_{i}\right)} f\left(x_{0}+z \frac{A_{i}}{\left\|A_{i}\right\|}+\sum_{k} y_{k} v_{k}\right) d y d z \leq h\left(b+\delta b_{i}\right)-h(b), \\
& \int_{0}^{\delta b_{i} /\left\|A_{i}\right\|} \int_{\Delta^{\prime 1}\left(\delta b_{i}\right)} f\left(x_{0}+z \frac{A_{i}}{\left\|A_{i}\right\|}+\sum_{k} y_{k} v_{k}\right) d y d z \geq h\left(b+\delta b_{i}\right)-h(b) .
\end{aligned}
$$

$f$ being continuously differentiable, one may write

$$
\begin{aligned}
f\left(x_{0}\right. & \left.+\sum_{k} y_{k} v_{k}+z \frac{A_{i}}{\left\|A_{i}\right\|}\right) \\
& =f\left(x_{0}+\sum_{k} y_{k} v_{k}\right)+z\left\langle\nabla f\left(x_{0}+\sum_{k} y_{k} v_{k}+\theta \frac{A_{i}}{\left\|A_{i}\right\|}\right), \frac{A_{i}}{\left\|A_{i}\right\|}\right\rangle
\end{aligned}
$$

for some $0<\theta<z$. Therefore, $\nabla f$ being bounded on a compact set, with a simple continuity argument we get

$$
\lim _{\delta b_{i} \rightarrow 0} \frac{h\left(b+\delta b_{i} e_{i}\right)-h(b)}{\delta b_{i}}=\frac{\int_{\Delta^{\prime 1}(0)} f\left(x_{0}+\sum_{k} y_{k} v_{k}\right) d y}{\left\|A_{i}\right\|}=\frac{\int_{\Omega_{i}} f d \mu}{\left\|A_{i}\right\|} .
$$

For $f$ not necessarily nonnegative, simply use the same argument with $(f+M)-$ $M$, where $\sup _{x \in \Omega}|f(x)| \leq M$ (as $f$ is continuous and $\Omega$ is compact).

Finally, the same argument also holds if $\delta b_{i}<0$, and the continuity of the partial derivatives is immediate from (2.3).

Remark 2.3. We have not used that $f$ is (positively) homogeneous, so Lemma 2.2 is valid for any continuously differentiable function $f$. In addition, note that if $\Omega_{i}=\emptyset$, then $\partial h(b) / \partial b_{i}=0$, in accordance with $0=\int_{\Omega_{i}} f d \mu$. Indeed, the constraint $A_{i}^{T} x \leq$ $b_{i}$ is strictly redundant and remains strictly redundant with a slight perturbation of $b_{i}$.

Theorem 2.4. Assume that $f$ is continuously differentiable, $\mathcal{V}_{n}(\Omega) \neq 0$, and, for all $i=1, \ldots, m, \mathcal{V}_{n-1}\left(\Omega_{i}\right) \neq 0$. Then

$$
\int_{\Omega} f(x) d x=\frac{1}{n+q} \sum_{i=1}^{m} \frac{b_{i}}{\left\|A_{i}\right\|} \int_{\Omega_{i}} f d \mu=\sum_{i=1}^{m} \frac{d\left(o, \mathcal{H}_{i}\right)}{n+q} \int_{\Omega_{i}} f d \mu,
$$

where $\mu$ is the Lebesgue measure on the $(n-1)$-dimensional affine variety $\mathcal{H}_{i}$ that contains $\Omega_{i}$. 
Proof. Since $h(b)$ is an homogeneous continuously differentiable function at $b$, by Euler's formula (2.1), one gets

$$
(n+q) h(b)=\langle\nabla h(b), b\rangle,
$$

which, using Proposition 2.1 and Lemma 2.2 for $\nabla h(b)$, yields (2.6).

Remark 2.5. (a) Formula (2.6) also holds if $\Omega_{i}=\emptyset$ for some $i$ 's. For such $i$ 's, $\int_{\Omega_{i}} f d \mu=0$, in accordance with $\partial h(b) / \partial b_{i}=0$ (cf. Remark 2.3).

(b) Note that the proof of Theorem 2.4 only uses Euler's formula. An alternative proof is to use Green's formula, i.e., with notation as in Prop. 2.3 , p. 128 in [6],

$$
\int_{\Omega} \operatorname{div}(X) f d \omega+\int_{\Omega} X f d \omega=\int_{\partial \Omega}\langle X, \vec{n}\rangle f d \sigma
$$

where $\vec{n}$ is the unit outward-pointing normal to $\partial \Omega$, and with the vector field $X:=\sum_{i=1}^{n} x_{i} \partial / \partial x_{i}$.

Hence, the integration of $f$ on $\Omega$ reduces to a weighted integration of $f$ on the $(n-1)$-dimensional faces of $\Omega$ (and in fact, only on those faces that do not contain the origin). A similar formula has already been given for $f:=e^{\langle c, x\rangle}$, using Stokes' formula (see [1], [2]).

For instance, if $P$ (resp. $Q$ ) is an homogeneous polynomial of degree $p$ (resp. $q$ ), then

$$
\int_{\Omega}(P+Q) d x=\sum_{i} d\left(o, \mathcal{H}_{i}\right) \int_{\Omega_{i}}\left(\frac{P}{n+p}+\frac{Q}{n+q}\right) d \mu .
$$

With $f \equiv 1$, one retrieves the volume formula given in [4] that is interpreted as a standard result in geometry. Indeed, in (2.6) $\int_{\Omega_{i}} f d \mu$ reduces to $\mathcal{V}_{n-1}\left(\Omega_{i}\right)$, the $(n-1)$-dimensional volume of $\Omega_{i}$, so that $b_{i} /\left(n\left\|A_{i}\right\|\right) \times \mathcal{V}_{n-1}\left(\Omega_{i}\right)$ is simply the $n$-dimensional version of the standard formula for the area of a triangle (base $\times$ height/2) and (2.6) reads

$$
\mathcal{V}_{n}(\Omega)=n^{-1} \sum_{i=1}^{m} \frac{b_{i}}{\left\|A_{i}\right\|} \mathcal{V}_{n-1}\left(\Omega_{i}\right)
$$

In [4], an algorithm based on (2.8) has been proposed, and the interested reader is referred to [3] for a numerical comparison of several algorithms for exact volume computation, including that one.

Remark 2.6. In fact Theorem 2.4 is also valid at points $b$ where $\mathcal{V}_{n-1}\left(\Omega_{i}\right)=0$ for some $i \in I \subset\{1, \ldots, m\}$. Indeed, one may prove that the constraint $A_{i}^{T} x \leq b_{i}, i \in I$, is redundant and therefore can be removed, i.e. $\Omega \equiv\left\{x \mid A_{i}^{T} x \leq b_{i}, i \notin I\right\}$. After having removed all the redundant constraints, (2.6) is valid, with the summation being now over all $i \notin I$. But (2.6) is also valid if we maintain those $i \in I$, since

$$
\mathcal{V}_{n-1}\left(\Omega_{i}\right)=0 \Rightarrow \mu\left(\Omega_{i}\right)=0 \Rightarrow \int_{\Omega_{i}} f d \mu=0 .
$$

2.1. Further results. We now would like to apply the same technique to $\int_{\Omega_{i}} f d \mu$ so as to consider integration on faces of lower dimensions. Indeed, we can do so provided $f$ has continuous second derivatives.

Let $b^{i}$ be the $(m-1)$-vector obtained from $b$ by deleting its ith entry, and let $A^{(i)}$ be the matrix obtained from $A$ by deleting its $i$ th row. Let $\left\{v_{k}\right\}$ be $n-1$ orthonormal vectors in the vector space associated with $\mathcal{H}_{i}$. For every $j \neq i$, let 
$B_{j}$ be the $(n-1)$-vector $\left\{B_{j k}\right\}$ with $B_{j k}:=A_{j}^{T} v_{k}, k=1, \ldots, n-1$, and with $x_{0}$ arbitrary, define

$$
\Gamma_{i}:=\left\{y \in R^{n-1} \mid B_{j}^{T} y \leq b_{j}-A_{j}^{T} x_{0}, j \neq i\right\}=\left\{y \in R^{n-1} \mid B y \leq b^{i}-A^{(i)} x_{0}\right\}
$$

and

$$
h\left(b^{i}, x_{0}\right):=\int_{B y \leq b^{i}-A^{(i)} x_{0}} f\left(x_{0}+\sum_{k=1}^{n-1} y_{k} v_{k}\right) d y .
$$

If $x_{0} \in \mathcal{H}_{i}$, then $\Gamma_{i}$ is the representation of $\Omega_{i}$ in an orthonormal basis of $\mathcal{H}_{i}$, and $h\left(b^{i}, x_{0}\right)=\int_{\Omega_{i}} f d \mu$, with $\mu$ the Lebesgue measure on $\mathcal{H}_{i}$. Finally, let

$$
\Omega_{i j}:=\left\{x \in \Omega \mid A_{i}^{T} x=b_{i}, A_{j}^{T} x=b_{j}\right\}
$$

be the $(i, j)\left((n-2)\right.$-dimensional) face of $\Omega$ and $\mathcal{H}_{i j}$ the $(n-2)$-dimensional affine variety that contains $\Omega_{i j}$.

Theorem 2.7. Let $f$ be twice continuously differentiable. Assume also that for every $i=1, \ldots, m$, either $\Omega_{i}=\emptyset$ or $\mathcal{V}_{n-1}\left(\Omega_{i}\right) \neq 0$, and for every $j=1, \ldots, m$ with $j \neq i$, either $\Omega_{i j}=\emptyset$ or $\mathcal{V}_{n-2}\left(\Omega_{i j}\right) \neq 0$. Then:

(a) $h\left(b^{i}, x_{0}\right)$ is positively homogeneous of degree $n+q-1$.

(b) With $x_{0} \in \mathcal{H}_{i}$ fixed, arbitrary,

$$
\begin{aligned}
\frac{\partial h\left(b^{i}, x_{0}\right)}{\partial b_{j}} & =\frac{1}{\left\|B_{j}\right\|} \int_{\Omega_{i j}} f d \nu, j \neq i, \\
\frac{\partial h\left(b^{i}, x_{0}\right)}{\partial x_{0 k}} & =\sum_{j \neq i} \frac{-A_{j k}}{\left\|B_{j}\right\|} \int_{\Omega_{i j}} f d \nu+\int_{\Omega_{i}} \frac{\partial f}{\partial x_{k}} d \mu,
\end{aligned}
$$

with $\mu$ (resp. $\nu$ ) the Lebesgue measure on $\mathcal{H}_{i}$ (resp. $\mathcal{H}_{i j}$ ).

(c) With $x_{0} \in \mathcal{H}_{i}$ fixed, arbitrary,

$$
\int_{\Omega_{i}} f d \mu=\frac{1}{n+q-1}\left[\sum_{j \neq i} d_{i}\left(x_{0}, \mathcal{H}_{i j}\right) \int_{\Omega_{i j}} f d \nu+\int_{\Omega_{i}}\left\langle\nabla f, x_{0}\right\rangle d \mu\right],
$$

with $d_{i}$ the algebraic (Euclidean) distance in $\mathcal{H}_{i}$.

Proof. (a) From the definition of $h\left(b^{i}, x_{0}\right)$ in $(2.9)$, we get

$$
\begin{aligned}
h\left(\lambda b^{i}, \lambda x_{0}\right) & =\int_{B y \leq \lambda\left(b^{i}-A^{(i)} x_{0}\right)} f\left(\lambda x_{0}+\sum_{k=1}^{n-1} y_{k} v_{k}\right) d y \\
& =\int_{B(y / \lambda) \leq b^{i}-A^{(i)} x_{0}} \lambda^{q} f\left(x_{0}+\sum_{k=1}^{n-1}\left(y_{k} / \lambda\right) v_{k}\right) \lambda^{n-1} d(y / \lambda) \\
& =\lambda^{n+q-1} \int_{B y \leq b^{i}-A^{(i)} x_{0}} f\left(x_{0}+\sum_{k=1}^{n-1} y_{k} v_{k}\right) d y \\
& =\lambda^{n+q-1} h\left(b^{i}, x_{0}\right) .
\end{aligned}
$$

(b) If $\Omega_{i}=\emptyset$, then $\Omega_{i j}=\emptyset$ as well, and $\int_{\Omega_{i j}} f d \nu=0$. Any slight perturbation of $b_{j}, j \neq i$, leaves $\Omega_{i}$ empty, so that $\partial h\left(b^{i}, x_{0}\right) / \partial b_{j}=0$, and thus (2.10) holds.

Assume now that $\mathcal{V}_{n-1}\left(\Omega_{i}\right) \neq 0$. If $\Omega_{i j}=\emptyset$, it remains empty for every sufficiently small perturbation of $b_{j}$, and therefore, $\Omega_{i}$ remains unchanged. Hence, $\left.\partial h\left(b^{i}, x_{0}\right) / \partial b_{j}\right)=0$, in accordance with $\int_{\Omega_{i j}} f d \nu=0$, i.e. (2.10) holds. 
Consider now the case where $\Omega_{i j} \neq \emptyset$ and write $h\left(b^{i}, x_{0}\right)$ as $G(\hat{b})=\int_{B y \leq \hat{b}} g(y) d y$, with

$$
\hat{b}:=b^{i}-A^{(i)} x_{0} \text { and } g(y):=f\left(x_{0}+\sum_{k=1}^{n-1} y_{k} v_{k}\right) .
$$

We can also write $B y \leq \hat{b}$ as

$$
B_{j}^{T} y \leq \hat{b}_{j}:=b_{j}-A_{j}^{T} x_{0} \text { for all } j \neq i .
$$

Applying Lemma 2.2 to $G$ (in Lemma 2.2, we did not use that $f$ was positively homogeneous, cf. Remark 2.3), we see that $G$ is continuously differentiable, and

$$
\frac{\partial h\left(b^{i}, x_{0}\right)}{\partial b_{j}}=\frac{\partial G(\hat{b})}{\partial \hat{b}_{j}}=\frac{1}{\left\|B_{i}\right\|} \int_{B y \leq \hat{b}, B_{j}^{T} y=\hat{b}_{j}} g d \nu,
$$

where $\nu$ is now the Lebesgue measure on the $(n-2)$-dimensional affine variety $\mathcal{H}_{i j} \subset \mathcal{H}_{i}$, that contains the polytope

$$
\left\{y \in R^{n-1} \mid B y \leq \hat{b}, B_{j}^{T} y=\hat{b}_{j}\right\}=\Omega_{i j} .
$$

This yields (2.10). To get (2.11), let $x_{0}:=x_{0}+\lambda e_{k}$ with $e_{k}$ the $n$-vector $\left\{\delta_{k j}\right\}$ (and $\delta_{k j}$ the Kronecker symbol). Then

$$
h\left(b^{i}, x_{0}+\lambda e_{k}\right)=\int_{B y \leq b^{i}-A^{(i)}\left(x_{0}+\lambda e_{k}\right)} f\left(x_{0}+\lambda e_{k}+\sum_{s=1}^{n-1} y_{s} v_{s}\right) d y .
$$

Define

$$
\Omega_{i}(\lambda):=\left\{y \in R^{n-1} \mid B y \leq b^{i}-A^{(i)} x_{0}-\lambda A^{(i)} e_{k}\right\} \text { and } \Omega_{i}(0)=\Omega_{i} .
$$

Now, writing $x^{\prime}:=x_{0}+\sum_{s=1}^{n-1} y_{s} v_{s}$, with $f$ twice continuously differentiable, we get

$$
f\left(x_{0}+\lambda e_{k}+\sum_{s=1}^{n-1} y_{s} v_{s}\right)=f\left(x^{\prime}\right)+\lambda \frac{\partial f\left(x^{\prime}\right)}{\partial x_{k}}+\lambda^{2} \frac{\partial^{2} f\left(x^{\prime}+\theta e_{k}\right)}{\partial x_{k}^{2}}
$$

for some $0<\theta<\lambda$. Hence,

$$
\begin{aligned}
\lambda^{-1}\left(h\left(b^{i}, x_{0}+\lambda e_{k}\right)-h\left(b^{i}, x_{0}\right)\right) & =\lambda^{-1}\left[\int_{\Omega_{i}(\lambda)} f\left(x^{\prime}\right) d y-\int_{\Omega_{i}} f\left(x^{\prime}\right) d y\right] \\
& +\int_{\Omega_{i}(\lambda)} \frac{\partial f\left(x^{\prime}\right)}{\partial x_{k}}+\lambda \frac{\partial^{2} f\left(x^{\prime}+\theta e_{k}\right)}{\partial x_{k}^{2}} d y
\end{aligned}
$$

As $f$ is twice continuously differentiable, $\left(\partial^{2} f\left(x^{\prime}\right) / \partial x_{k}^{2}\right)$ is bounded on a compact set. In addition, for $\lambda$ sufficiently small, $\Omega_{i}(\lambda)$ is contained in some compact set. Therefore, in the above equation, the term $\lambda \int_{\Omega_{i}(\lambda)}\left(\partial^{2} f\left(x^{\prime}+\theta e_{k}\right) / \partial x_{k}^{2}\right) d y$ vanishes as $\lambda \rightarrow 0$.

In addition, by a simple continuity argument,

$$
\lambda \rightarrow 0 \Rightarrow \int_{\Omega_{i}(\lambda)} \frac{\partial f\left(x^{\prime}\right)}{\partial x_{k}} d y \rightarrow \int_{\Omega_{i}(0)} \frac{\partial f\left(x^{\prime}\right)}{\partial x_{k}} d y=\int_{\Omega_{i}} \frac{\partial f}{\partial x_{k}} d \mu,
$$

with $\mu$ the Lebesgue measure on $\mathcal{H}_{i}$.

Finally, write

$$
g(y):=f\left(x_{0}+\sum_{s=1}^{n-1} y_{s} v_{s}\right) \text { and } \hat{b}_{j}(\lambda):=b_{j}-A_{j}^{T} x_{0}-\lambda A_{j k}, j \neq i .
$$


Denote

$$
G(\hat{b}(\lambda)):=\int_{\Omega_{i}(\lambda)} f\left(x^{\prime}\right) d y=\int_{B y \leq \hat{b}(\lambda)} g(y) d y .
$$

Assume first that $\mathcal{V}_{n-2}\left(\Omega_{i j}\right) \neq 0$. Again, we can apply Lemma 2.2 to $G$, since $g$ is continuously differentiable and $\mathcal{V}_{n-2}\left(\Omega_{i j}\right) \neq 0$. Therefore, one gets

$$
\frac{\partial G(\hat{b})}{\partial \hat{b}_{j}}=\frac{1}{\left\|B_{j}\right\|} \int_{B y \leq \hat{b}, B_{j}^{T} y=\hat{b}_{j}} g d \nu=\frac{1}{\left\|B_{j}\right\|} \int_{B y \leq \hat{b}, B_{j}^{T} y=\hat{b}_{j}} f d \nu,
$$

with $\nu$ the Lebesgue measure on the $(n-2)$-dimensional affine variety $\mathcal{H}_{i j} \subset \mathcal{H}_{i}$ that contains the convex polytope $\left\{y \in R^{n-1} \mid B y \leq \hat{b}, B_{j}^{T} y=\hat{b}_{j}\right\}=\Omega_{i j}$. Hence, from

$$
\lim _{\lambda \rightarrow 0} \lambda^{-1}\left(\int_{\Omega_{i}(\lambda)} f\left(x^{\prime}\right) d y-\int_{\Omega_{i}} f\left(x^{\prime}\right) d y\right)=\sum_{j \neq i} \frac{\partial G(\hat{b}(0))}{\partial \hat{b}_{j}} \frac{d \hat{b}_{j}(0)}{d \lambda}
$$

and $d \hat{b}_{j} / d \lambda=-A_{j k}$, one gets

$$
\lim _{\lambda \rightarrow 0} \lambda^{-1}\left(\int_{\Omega_{i}(\lambda)} f\left(x^{\prime}\right) d y-\int_{\Omega_{i}} f\left(x^{\prime}\right) d y\right)=\sum_{j \neq i} \frac{-A_{j k}}{\left\|B_{j}\right\|} \int_{\Omega_{i j}} f d \nu .
$$

If $\Omega_{i j}=\emptyset$, then $\Omega_{i}(\lambda)=\Omega_{i}$ for $\lambda$ sufficiently small, and therefore,

$$
\lim _{\lambda \rightarrow 0} \lambda^{-1}\left[\int_{\Omega_{i}(\lambda)} g(y) d y-\int_{\Omega_{i}} g(y) d y\right]=0,
$$

in accordance with $\int_{\Omega_{i j}} f d \nu=0$. Finally, combining (2.13) and (2.14) yields (2.11).

(c) To get (2.12), we just apply Euler's formula $(2.1)$ to $h\left(b^{i}, x_{0}\right)$, which is positively homogeneous of degree $n+q-1$, and continuously differentiable. This yields

$$
\begin{aligned}
\int_{\Omega_{i}} f d \mu & =h\left(b^{i}, x_{0}\right)=\frac{1}{n+q-1}\left\langle\nabla h\left(b^{i}, x_{0}\right),\left(b^{i}, x_{0}\right)\right\rangle \\
& =\frac{1}{n+q-1}\left[\left\langle\nabla_{b^{i}} h\left(b^{i}, x_{0}\right), b^{i}\right\rangle+\left\langle\nabla_{x_{0}} h\left(b^{i}, x_{0}\right), x_{0}\right\rangle\right] .
\end{aligned}
$$

Using (2.10)-(2.11) for $\nabla h\left(b^{i}, x_{0}\right)$ in the above expression, one gets

$$
\int_{\Omega_{i}} f d \mu=\frac{1}{n+q-1}\left[\sum_{j \neq i} \frac{b_{j}-A_{j}^{T} x_{0}}{\left\|B_{j}\right\|} \int_{\Omega_{i j}} f d \nu+\int_{\Omega_{i}}\left\langle\nabla f, x_{0}\right\rangle d \mu\right] .
$$

Noting that $\left(b_{j}-A_{j}^{T} x_{0}\right) /\left\|B_{j}\right\|$ is just $d_{i}\left(x_{0}, \mathcal{H}_{i j}\right)$ (the algebraic distance in $\mathcal{H}_{i}$ from the origin $x_{0}$ to $\left.\mathcal{H}_{i j}\right)$, one gets $(2.12)$.

Hence, integrating $f$ on $\Omega$ reduces to

- either integrating $f$ on the $(n-1)$-dimensional faces of $\Omega$ (cf. Theorem 2.4),

- or integrating $f$ on the $(n-2)$-dimensional faces of $\Omega$ and its derivatives on the $(n-1)$-dimensional faces of $\Omega$ (cf. Theorem 2.7).

Provided $f$ has continuous partial derivatives of order $p+1$, one may iterate the above procedure and show that it suffices to evaluate $f$ and its first, second, $\ldots, p$ th derivatives at the vertices of $\Omega$, the (1)-dimensional faces, etc. 
For instance, consider the term $\int_{\Omega_{i}}\left\langle\nabla f, x_{0}\right\rangle d \mu$. Let $z_{0} \in \mathcal{H}_{i}$ be arbitrary, and with the same notation as in the proof of Theorem 2.7, write

$$
g\left(b^{i}, z_{0}\right):=\int_{\Omega_{i}}\left\langle\nabla f, x_{0}\right\rangle d \mu=\int_{B y \leq b^{i}-A^{(i)} z_{0}}\left\langle\nabla f\left(z_{0}+\sum_{k=1}^{n-1} y_{k} v_{k}\right), x_{0}\right\rangle d y .
$$

Again, $g$ is (positively) homogeneous of degree $(n+q-2)$ since $\nabla f$ is positively homogeneous of degree $q-1$. Therefore, if $f$ has continuous third derivatives, proceeding with similar arguments as in the proof of Theorem 2.7, one gets:

$$
\int_{\Omega_{i}}\left\langle\nabla f, x_{0}\right\rangle d \mu=\frac{1}{n+q-2}\left[\sum_{j \neq i} \frac{d_{i}\left(z_{0}, \mathcal{H}_{i j}\right)}{\left\|B_{j}\right\|} \int_{\Omega_{i j}}\left\langle\nabla f, x_{0}\right\rangle d \nu+\int_{\Omega_{i}}\left\langle z_{0},\left(\partial^{2} f\right) x_{0}\right\rangle d \mu\right],
$$

with $\partial^{2} f$ the Hessian matrix of $f$.

An interesting case is when $f$ is an homogeneous polynomial of degree $q$. Then the $(q+1)$ th derivatives vanish, and integrating that polynomial on $\Omega$ requires only knowledge of the polynomial and all its partial derivatives at the vertices of $\Omega$, i.e. at a finite number of points. As a continuous function on a compact set can be approximated by polynomials (a sum of homogeneous polynomials), one may compute a good approximation of the integral by considering only the vertices of $\Omega$.

Finally, one may notice that integration on a nonconvex polytope reduces to the above case after a partition of the original polytope into convex polytopes.

2.2. Illustrative example. In $R^{2}$, consider $J:=\int_{\Omega} x y d x d y$ with

$$
\Omega:=\left\{(x, y) \in R^{2} \mid x+y \leq 1, x \geq a, y \geq b\right\},
$$

i.e. $n=q=2$. A direct integration yields

$$
J=\frac{1}{8}\left[(1-b)^{4}-a^{4}\right]-\frac{1}{3}\left[(1-b)^{3}-a^{3}\right]+\frac{1}{4}\left(1-b^{2}\right)\left[(1-b)^{2}-a^{2}\right] .
$$

Now, with $\Omega_{1}:=\Omega \cap\{x=a\}$, we get

$$
d\left(o, \mathcal{H}_{1}\right) \int_{\Omega_{1}} f d \mu=-a \int_{b}^{1-a} a v d v=-a^{2}\left[(1-a)^{2}-b^{2}\right] / 2 .
$$

With $\Omega_{2}:=\Omega \cap\{y=b\}$, we get

$$
d\left(o, \mathcal{H}_{2}\right) \int_{\Omega_{2}} f d \mu=-b \int_{a}^{1-b} b v d v=-b^{2}\left[(1-b)^{2}-a^{2}\right] / 2 .
$$

With $\Omega_{3}:=\Omega \cap\{x+y=1\}$, we get

$$
d\left(o, \mathcal{H}_{3}\right) \int_{\Omega_{3}} f d \mu=\frac{1}{\sqrt{2}} \int_{a}^{1-b} \sqrt{2} v(1-v) d v=\frac{1}{2}\left[(1-b)^{2}-a^{2}\right]-\frac{1}{3}\left[(1-b)^{3}-a^{3}\right]
$$

and one may check that

i.e.,

$$
J=\frac{1}{4}\left[-a^{2} \int_{b}^{1-a} v d v-b^{2} \int_{a}^{1-b} v d v+\int_{a}^{1-b} v(1-v) d v\right]
$$

$$
J=\frac{1}{4} \sum_{i=1}^{3} d\left(0, \mathcal{H}_{i}\right) \int_{\Omega_{i}} f d \mu
$$

or equivalently, (2.6) is satisfied. 
Similarly, take $x_{0}:=(1-b, b) \in \mathcal{H}_{3}$. Then

$$
d_{3}\left(x_{0}, \mathcal{H}_{2}\right)=0, d_{3}\left(x_{0}, \mathcal{H}_{1}\right)=\sqrt{2}(1-a-b), f((a, 1-a))=a(1-a) .
$$

In addition,

$$
\int_{\Omega_{3}}\left\langle\nabla f(x), x_{0}\right\rangle \mu(d x)=\sqrt{2} \int_{b}^{1-a}[v(1-b)+(1-v) b] d v
$$

and one may check that

$$
\frac{\sqrt{2}}{3}\left[(1-a-b) a(1-a)+\int_{b}^{1-a}[v(1-b)+b(1-v)] d v\right]=\int_{\Omega_{3}} f d \mu,
$$

i.e. (2.12) is satisfied.

\section{REFERENCES}

1. A. Barvinok, Computing the volume, counting integral points, and exponential sums, Discrete \& Computational Geometry 10 (1993), pp. 123-141. MR 94d:52005

2. M. Brion, Points entiers dans les polydres convexes, Ann. Sci. Ec. Norm. Sup., Série IV, 21 (1988), pp. 653-663. MR 90d:52020

3. B. Beeler, A. Enge, K. Fukuda, H-J. Lthi, Exact volume computation for polytopes: a practical study, 12th European Workshop on Computational Geometry, Muenster, Germany, March 1996.

4. J.B. Lasserre, An analytical expression and an algorithm for the volume of a convex polyhedron in $R^{n}$, J. Optim. Theor. Appl. 39 (1983), pp. 363-377. MR 84m:52018

5. G. Cagnac, E.Ramis, J. Commeau, Analyse, Masson, Paris, 1970.

6. M.E. Taylor, Partial Differential Equations: Basic Theory, Springer-Verlag, New York, 1996. CMP 96:14

LAAS-CNRS, 7 Avenue du Colonel Roche, 31077 Toulouse Cédex 4, France

E-mail address: lasserre@laas.fr 